\title{
The Prognostic Role of TNM Staging Compared With Tumor Volume and Number of Pleural Sites in Malignant Pleural Mesothelioma
}

\author{
Claudia Proto, ${ }^{1}$ Diego Signorelli, ${ }^{1}$ Sandra Mallone, ${ }^{2}$ Arsela Prelaj, \\ Giuseppe Lo Russo, 'Martina Imbimbo, ${ }^{1}$ Giulia Galli, ${ }^{1}$ Roberto Ferrara, \\ Monica Ganzinelli, ${ }^{1}$ Giovanni Leuzzi, ${ }^{3}$ Francesca Gabriella Greco, ${ }^{4}$ \\ Giuseppina Calareso, ${ }^{4}$ Laura Botta, ${ }^{5}$ Gemma Gatta, ${ }^{5}$ Marina Garassino, ${ }^{1}$ \\ Annalisa Trama ${ }^{5}$
}

\begin{abstract}
Malignant pleural mesothelioma (MPM) is an aggressive disease with a unique morphology and distribution. Because of its peculiar growth pattern, clinical staging is difficult. Quantitative assessment such as tumor volume (TV) was suggested as an alternative prognostic evaluation. In this study we aimed to compare the prognostic role of Tumor, Node, Metastases (TNM) clinical staging with that of alternative staging approaches on the basis of the use of 2 quantitative clinical parameters, TV and number of pleural sites (NPS), in MPM patients (pts). Our data confirmed the prognostic role of TNM, tumor size, TV, and NPS. However, the TV and NPS combination performed better than TV, NPS, and TNM alone as prognostic classifier. Considering different quantitative parameters and translating such an approach from research level to clinical practice could increase prognostic accuracy for MPM pts, and help clinicians choose the best therapeutic strategy. Background: Age, sex, stage, histotype, and surgery are the most recognized prognostic factors for malignant pleural mesothelioma (MPM). Tumor volume (TV) was suggested as an alternative prognostic evaluation. We aimed to assess the prognostic role of Tumor, Node, Metastases (TNM) versus TV and number of pleural sites (NPS). Patients and Methods: Information on stage, TV, and NPS was collected for 52 MPM patients (pts) at our institution from 2009 to 2012. Baseline computed tomography imaging was performed to define TNM, TV, and NPS. Pts were divided in 3 stage groups: early (I-II), III, and IV. A dedicated computer system calculated TV. Pts were divided in 2 groups according to mean baseline TV $\left(483 \mathrm{~cm}^{3}\right)$. NPS was defined on the basis of the NPS macroscopically involved by disease (1-3). The association between TNM, tumor size (T), TV, NPS, TV and NPS, and overall survival was assessed using Cox models adjusted for age, sex, histology, and treatment. Results: Most pts were male; mean age was 62 years. We showed an association between TV, TNM, and T. Stage III (hazard ratio [HR], 4.71; $P=.02$ ) and IV (HR, 7.40; $P<.01)$, T3 (HR, 5.07; $P<.01)$ and T4 (HR, 5.09; $P<.01)$ TV $>483 \mathrm{~cm}^{3}(\mathrm{HR}, 3.47 ; P<.01)$ and NPS $2(\mathrm{HR}, 3.00 ; P=.08)$ and 3 (HR, 6.05; $P<.01$ ) were predictive of worse survival. However, the TV and NPS combination performed better than TV, NPS, and TNM alone as a prognostic classifier. Conclusion: We showed that TV is related to TNM staging and T, in particular. Improved prognostic performance might be achievable using quantitative clinical staging combining TV and NPS.
\end{abstract}

Clinical Lung Cancer, Vol. 20, No. 6, e652-60 ๑ 2019 Elsevier Inc. All rights reserved.

Keywords: Malignant pleural mesothelioma, Number of pleural sites, Prognosis, TNM, Tumor volume

C.P. and D.S. contributed equally to this work as first authors.

M.G. and A.T. contributed equally to this work as last authors.

${ }^{1}$ Department of Medical Oncology, Fondazione IRCCS Istituto Nazionale dei Tumori di Milano, Milan, Italy

${ }^{2}$ Department of Oncology and Molecular Medicine, Istituto Superiore di Sanità, Rome, Italy

${ }^{3}$ Thoracic Surgery Unit
${ }^{4}$ Department of Radiology

${ }^{5}$ Department of Preventive and Predictive Medicine, Evaluative Epidemiology Unit, Fondazione IRCCS Istituto Nazionale dei Tumori di Milano, Milan, Italy

Submitted: Feb 22, 2019; Revised: Apr 25, 2019; Accepted: Jun 15, 2019; Epub: Jun 26, 2019

Address for correspondence: Claudia Proto, MD, Department of Medical Oncology, Fondazione IRCCS Istituto Nazionale dei Tumori di Milano, Via G. Venezian, 1, 20133 Milan, Italy

E-mail contact: claudia.proto@istitutotumori.mi.it 


\section{Introduction}

Malignant pleural mesothelioma (MPM) is a rare tumor characterized by a median overall survival (OS) of approximately 9 to 17 months. ${ }^{1}$ It is strongly associated with asbestos exposure and despite the asbestos ban in 55 countries, more than 100 million people worldwide continue to be exposed. ${ }^{2}$ Moreover, as a result of past exposure and the long latency period ${ }^{3}$ a peak of incidence is expected in developed countries by $2030 .{ }^{4}$

To date, stage, age, sex, histotype, and treatment are the most recognized prognostic factors in $\mathrm{MPM}^{5}$ and the Tumor, Node, Metastases (TNM) eighth edition is the standard worldwide staging system for MPM. ${ }^{6}$ Because of its wide availability and limited cost, computed tomography (CT) imaging ${ }^{7}$ is the primary imaging method to assess the extension of MPM for staging and follow-up during treatment. Magnetic resonance imaging can supplement CT imaging in determining diaphragmatic and thoracic invasion. ${ }^{8}$ Also, functional data from [18F]-fluorodeoxyglucose positron emissions tomography can be useful for preoperative staging of MPM patients (pts), to identify distant metastases and in distinguishing between malignant and benign lesions. ${ }^{8}$ Unfortunately, MPM has a unique morphology and distribution. Its peculiar growth pattern is characterized by not well separate lesions that extend from the pleural surface to the surrounding tissues and structures and show variable thickness in different, also adjacent areas. Thus, all of the available imaging techniques cannot define the real disease extension and so the clinical TNM staging is still inaccurate in MPM.

To tackle the complex morphology of MPM, an international panel of experts strongly advocated the development of a new robust and uniform clinical staging system suggesting that the 3-dimensional display of axial images could more accurately portray overall disease extent. ${ }^{9}$ Thus, the ability to stratify prognosis ${ }^{10-20}$ and/or to better define clinical staging ${ }^{21}$ of the full 3-dimensional tumor volume (TV), tumor size (T) measurement using CT imaging, has been widely investigated. With the objective to improve the clinical staging, the combination of the standard staging system with other quantitative factors, such as pleural thickness and diaphragmatic thickness, has been evaluated, showing significant correlation with survival for both quantitative parameters. ${ }^{22,23}$ Finally, Gill and colleagues reported that a new quantitative staging system, on the basis of the measurement of TV and the maximal interlobar disease thickness, could better define the MPM prognosis compared with clinical TNM staging. ${ }^{24}$ Against this background, we hypothesized that the number of macroscopically involved pleural sites (NPS), a new feature not previously assessed and easily evaluable using CT imaging, that reflects the aggressiveness of disease, could influence MPM patient prognosis. We therefore speculated that the use of 2 quantitative clinical parameters, TV and NPS, could be useful in clinical staging, increasing its prognostic role.

In this article we report the results of our study aimed to compare the prognostic role of TNM clinical staging with that of alternative staging approaches on the basis of the use of 2 quantitative clinical parameters, TV and NPS, in MPM pts.

\section{Patients and Methods}

At the National Cancer Institute of Milan (INT), we retrospectively retrieved 52 pts with histologically confirmed MPM between
Table 1 Demographic and Clinical Characteristics of the Population Studied

\begin{tabular}{|c|c|c|}
\hline Characteristic & $\mathbf{n}$ & $\%$ \\
\hline \multicolumn{3}{|l|}{ Sex } \\
\hline Male & 35 & 67 \\
\hline Female & 17 & 33 \\
\hline \multicolumn{3}{|l|}{ Age } \\
\hline$<65 \mathrm{Y}$ & 32 & 62 \\
\hline$\geq 65 Y$ & 20 & 38 \\
\hline \multicolumn{3}{|l|}{ Histology } \\
\hline Epithelioid & 44 & 85 \\
\hline Biphasic & 8 & 15 \\
\hline \multicolumn{3}{|l|}{ Stage } \\
\hline Early (I-II) stage & 6 & 12 \\
\hline Stage III & 20 & 38 \\
\hline Stage IV & 26 & 50 \\
\hline \multicolumn{3}{|l|}{$\begin{array}{l}\text { Tumor Volume } \\
\text { Average }\end{array}$} \\
\hline$\leq 483 \mathrm{~cm}^{3}$ & 36 & 69 \\
\hline$>483 \mathrm{~cm}^{3}$ & 16 & 31 \\
\hline \multicolumn{3}{|l|}{$\begin{array}{l}\text { Number of Involved } \\
\text { Pleura Sites }\end{array}$} \\
\hline 1 & 7 & 13 \\
\hline 2 & 14 & 27 \\
\hline 3 & 31 & 60 \\
\hline \multicolumn{3}{|l|}{ Surgery } \\
\hline Yes (EPP) & 14 & 27 \\
\hline Yes (PD+) & 9 & 17 \\
\hline No & 29 & 56 \\
\hline \multicolumn{3}{|l|}{ Chemotherapy } \\
\hline No & 13 & 25 \\
\hline Yes & 37 & 71 \\
\hline Missing information & 2 & 4 \\
\hline
\end{tabular}

Abbreviations: $\mathrm{EPP}=$ extrapleural pneumonectomy; $\mathrm{PD}+=$ pleurectomy/decortication.

2009 and 2012. The study was approved by the local institutional review board (INT 67_18) and conducted according to the ethical principles for medical research involving human subjects adopted in the Declaration of Helsinki. Information on age, sex, histology, stage, TV, NPS, treatment, and life status were collected. The availability of a baseline CT scan at the time of histological diagnosis was an essential requirement.

Two experienced, dedicated thoracic radiologists independently evaluated and reviewed the CT images to define staging, TV, and NPS parameters. The radiologists were blinded to scan origin, patient clinical and demographic information, and the assessments of the other radiologist. Contrast-enhanced CT scans were transferred to a Synge MultiModality Workplace (Siemens) and reviewed with a soft tissue kernel at a window window/level 300/ 40 Hounsfield unit (HU). Clinical stage was defined according to the TNM (seventh edition) staging system and pts were divided in 3 stage groups: early (including stage I-II), III, and IV. Gross TV was calculated by multiplying the sum of all the tumor areas by 


\section{Tumor Volume and Number of Pleural Sites in MPM}

\section{Figure 1 Relationship Between Tumor Volume (TV) With (A) Malignant Pleural Mesothelioma Stage and (B) Tumor Size (T)}

A

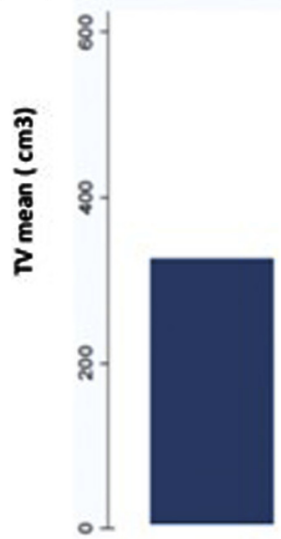

earlystage (1-II)

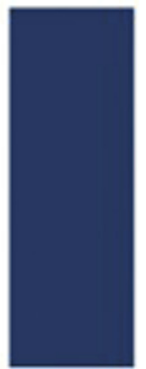

stage III

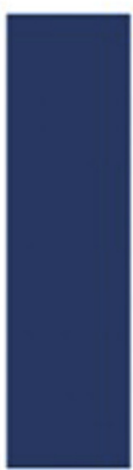

stage IV
B

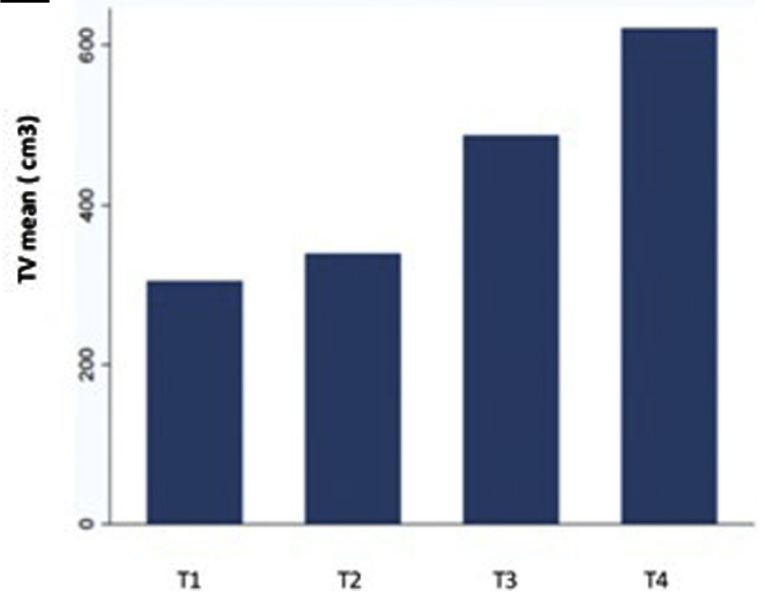

the section thicknesses. The pleural thickness was measured using the semiautomated software, while the radiologist indicates density's range (40-100 HU) and edges of the suspected lesions. After that, the software was used to calculate the value of the area and finally the entire process was revised by both radiologists to check any inaccuracies. Regarding TV, pts were divided in 2 groups on the basis of the mean value of baseline TV $\left(483 \mathrm{~cm}^{3}\right.$; range, $18-$ $\left.2329 \mathrm{~cm}^{3}\right)$ : pts with large TV $\left(>483 \mathrm{~cm}^{3}\right)$ and pts with small TV $\left(\leq 483 \mathrm{~cm}^{3}\right)$.

The NPS was defined according to the macroscopic disease extension to 1 or more of the 3 possible pleural surfaces: the mediastinal, diaphragmatic, and parietal surface. We classified NPS according to the number of the involved pleural surfaces: 1 site (1) if there was the exclusive extension to the parietal, mediastinal, or diaphragmatic pleura; 2 sites (2) if there was the concomitant involvement of 2 pleural sites (parietal and mediastinal or parietal and diaphragmatic or mediastinal and diaphragmatic); and 3 sites (3) if the tumor involved all of the 3 pleural surfaces. The macroscopic involvement of the pleural surface was defined by the radiologist as pleural thickening of at least $2 \mathrm{~mm}$ at CT imaging. Regarding treatment, we categorized pts for those who were not treated, those with missing information on treatment, and those who underwent respectively: only chemotherapy, only surgery, and chemotherapy and surgery.

\section{Statistical Analysis}

The relationships between TV, stage, and T according to TNM, were analyzed by comparing the mean TV in cubic centimeters across the different stages and $\mathrm{T}$ sizes.

Overall survival was defined as the time (in months) from diagnosis to death. Kaplan-Meier analyses and log rank test were performed for OS to determine significant prognostic factors (ie, $\mathrm{T}$, N, M; stage; TV; NPS; and clinical features [age, sex, histology, and treatment]). Association between stage (early stage [I-II], stage III, stage IV), $\mathrm{T}$ on the basis of the $\mathrm{T}$ parameter according to TNM definition (T1-T2; T3; T4), TV, NPS, and the combination of TV and NPS with OS was evaluated using 6 separate Cox models adjusted for age, sex, histology, and treatment. The effect of stage and $\mathrm{T}$ was tested separately on $\mathrm{OS}$ in models to avoid multicollinearity.

\section{Results}

Characteristics of pts included in our study are reported in Table 1 . Most of them, 35 pts (67\%) were male and 32 pts younger than 65 years old (62\%). Median age was 62 years. Almost all pts, 44 pts (85\%) had epithelioid histology and no pts showed a pure sarcomatoid histology. Half of the pts were diagnosed in stage IV and the remaining were mainly stage III; only 6 pts (12\%) were diagnosed in an early stage. According to the mean value of baseline TV, $69 \%$ of pts had a TV $>483 \mathrm{~cm}^{3}$ at baseline CT imaging and most of them had an involvement of all 3 pleural surfaces (60\%). Finally, approximately half of the pts received radical surgery with curative intent, $27 \%$ received extrapleural pneumonectomy (EPP) and $17 \%$ received pleurectomy and decortication $(\mathrm{P} / \mathrm{D})$. Chemotherapy was performed in $71 \%$ of pts. No major differences between pts who received radical surgery and those who did not were observed in terms of age, stage, and histology (Supplemental Table 1). No major differences were observed in terms of age, sex, stage, and histology between pts who underwent EPP, P/D, and those who did not (Supplemental Table 1). Also, for chemotherapy and treatment no significant differences were found in terms of different distribution of age, sex, stage, and histology (Supplemental Table 1). In Figure 1 the relationship between TV and the 3 defined stage groups (Figure 1A) and the relationship of TV and $\mathrm{T}$ as defined according to the T1 parameter of TNM are reported (Figure 1B). Our results show that the mean TV increases with the advancement of the stage (Figure 1A) and in pts with higher $\mathrm{T}$ (Figure 1B). 

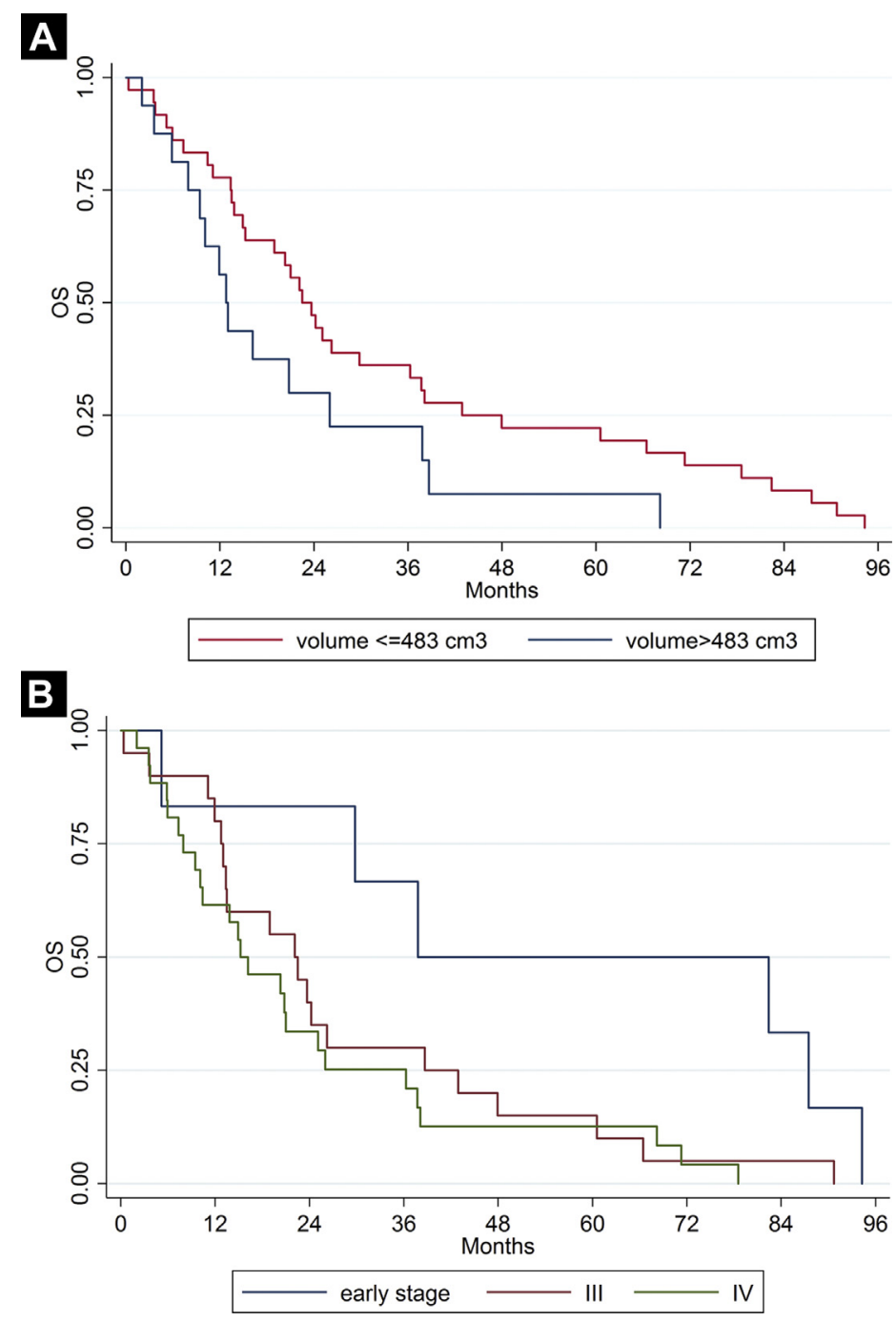

The survival analyses for OS according to TV and stage are reported in Figure 2. OS was higher for pts with TV $\leq 483 \mathrm{~cm}^{3}$ (Figure 2A) and for pts with early stage (Figure 2B).

Table 2 shows the Cox models used to assess the association between OS and stage grouping ( $\mathrm{B}$ model) and between OS and the $\mathrm{T}$ parameter ( $\mathrm{C}$ model). Moreover, in Table 3 the Cox models used to evaluate the association of TV groups (D model), of NPS groups (E model), and of the combination of TV groups and NPS groups (F model) with OS are reported.

Finally, we found a significant prognostic role on OS of clinical stage, T, TV, NPS, and combination of TV groups and NPS groups. In our study, stage III (hazard ratio [HR], 4.71; 95\% confidence interval [CI], 1.35-16.45; $P=.02)$ and IV (HR, 7.40; 95\% CI, 1.89-28.91; $P<.01)$ were associated with worse survival as well as higher $\mathrm{T}$ identified according to the $\mathrm{T}$ parameter: $\mathrm{T} 3$ (HR, 5.07; 95\% CI, 1.98-13.03; $P<.01$ ) and T4 (HR, 5.09; 95\% CI, 1.68-15.38; $P<.01)$. Pts with large TV were associated with worse survival (HR, 3.47; 95\% CI, 1.5-8.02; $P<.01)$ than pts with small TV. Pts with 2 (HR, 3.0; 95\% CI, 0.87-10.37; $P=.08$ ) and 3 macroscopically involved pleural surfaces (HR, 6.05; 95\% CI, 1.91-19.12; $P<.01$ ) had worse prognosis compared with pts with only 1 pleural surface involved on CT scan. However, the quantitative clinical staging comprising the combination of TV and NPS performed better as a prognostic classifier than TV, NPS, and TNM alone. Pts with small TV and 2 NPS had an HR of 8.16 (95\% CI, 1.97-33.87; $P<.01$ ), pts with large TV and 3 NPS had an HR of 17.76 (95\% CI, 3.44-91.8; $P<.01$ ). We can observe a progressive increase of HR combining a large TV with higher NPS. Pts with large TV and high NPS had the worst prognosis (Table 3).

\section{Discussion}

In our study we assessed the prognostic role of TV, NPS, and of the combination of TV groups and NPS groups supporting the importance of combining different quantitative parameters to increase the prognostic role of clinical staging in MPM. 


\section{Tumor Volume and Number of Pleural Sites in MPM}

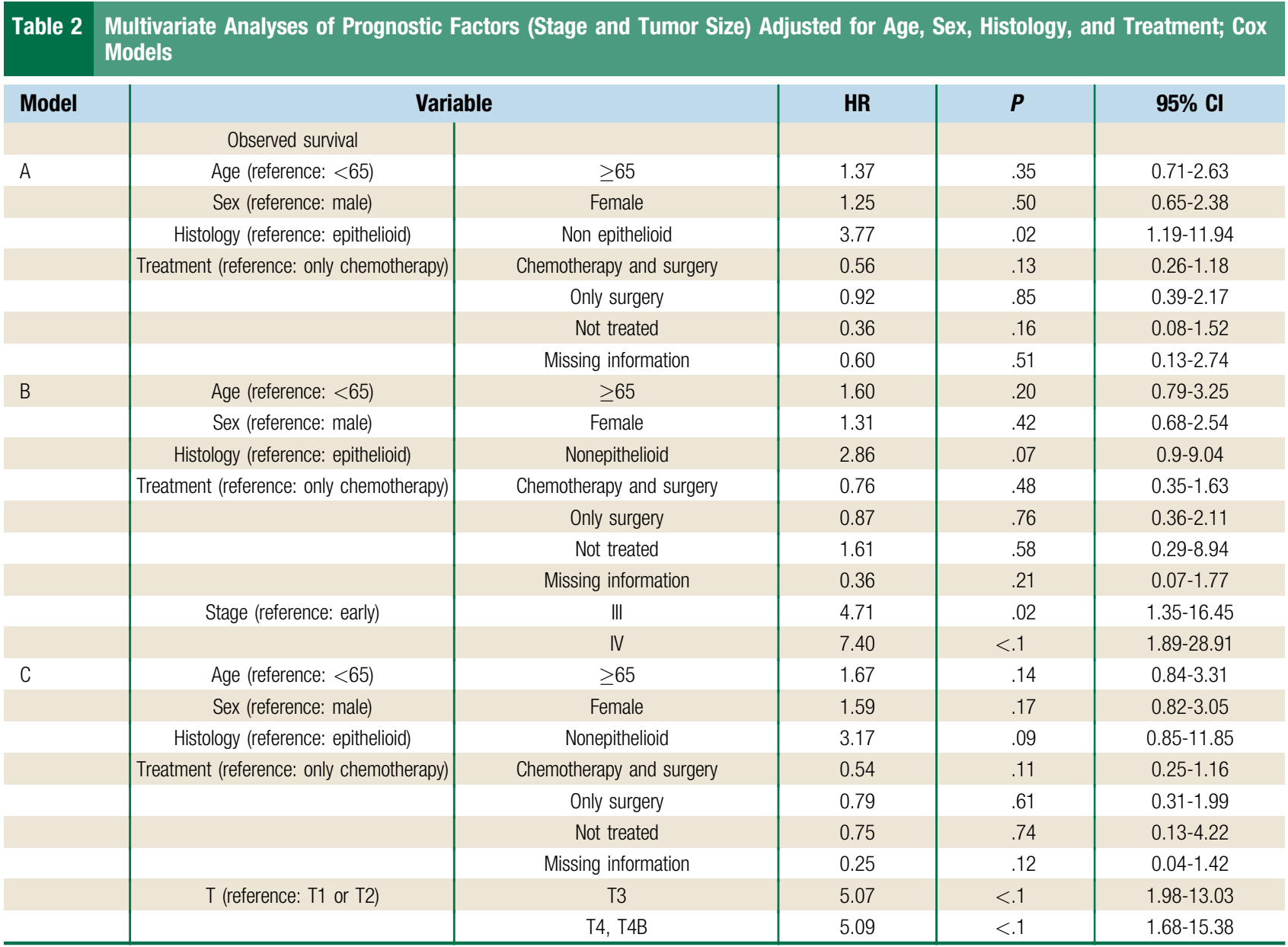

Abbreviation: $\mathrm{HR}=$ hazard ratio.

Our work confirms the previously reported association between $\mathrm{TV}$, stage, and clinical $\mathrm{T}^{21,25}$ as well as the prognostic role of TV. In detail, considering its effect on patient survival, TV alone showed a significant HR of 3.47. Several studies supported the role of TV at the time of CT imaging as a prognostic factor in the presurgical setting and as a parameter to evaluate the patient's treatment response. ${ }^{10-20}$ Rusch and colleagues, ${ }^{21}$ in a multicenter North American pilot study, showed the feasibility of incorporating the TV at the time of CT imaging in the clinical staging of 129 MPM pts who underwent surgery. They reported a correlation between TV and surgical pT, pN, and OS, with a trend toward a worse survival in pts with the largest TV $\left(\mathrm{TV}>511.3 \mathrm{~cm}^{3}\right)$. In particular, distinguishing pts in 3 different groups, according to the average TV $\left(91.2,245.3\right.$, and $\left.511.3 \mathrm{~cm}^{3}\right)$, they achieved a median OS of 37,18 , and 8 months in the 3 different prognostic groups, respectively. ${ }^{21}$

In our study, we showed that the combination of TV and NPS improved the prognostic prediction. The TV alone had an HR of 3.47; the NPS groups 2 and 3 had an HR of 3.00 and 6.05, respectively. The HRs of the combinations of TV and NPS ranged from 5.19 to 17.76 . In the literature, other quantitative radiological measures have been explored to improve the TNM system prognostic role. The pleural thickness measured on CT scan in 3 distinct sites of the thorax was investigated by the International Association for the Study of Lung Cancer/International Mesothelioma Interest Group group on a large series database. They showed a significant correlation between this quantitative parameter and $\mathrm{T}$ and $\mathrm{N}$ parameters of the TNM classification and survival. ${ }^{22}$

A second study showed an independent and significant prognostic role (HR, 1.12; 95\% CI, 1.02-1.23) of tumor thickness in 65 MPM pts who received preoperative radiotherapy. ${ }^{23}$ More recently, another group retrospectively investigated the characteristics at baseline CT imaging of 161 MPM pts, using a novel approach for the evaluation of $\mathrm{T}$ on the basis of the use of the maximal tumor thickness and the disease extension to the chest wall. In accordance with our results, in their analysis the estimation of $\mathrm{T}$ was a better prognostic factor than TNM stage and was related to the sarcomatoid histology and TNM stages; moreover, the prognostic role of tumor extension was higher than that of tumor thickness. ${ }^{26}$

Regarding the comparison with stage, we found that the combinations of TV groups and NPS groups had higher HRs than stage. These results are in line with other studies. In the study of Gill et al, the accuracy of the TNM clinical staging was compared with 2 potential quantitative parameters (TV and maximal thickness of disease in the interlobar fissures) in 472 MPM pts who underwent surgery at a single institution. The aim was the identification of a new model able to improve the pretreatment 


\begin{tabular}{|c|c|c|c|c|c|}
\hline \multirow[t]{2}{*}{ Model } & \multicolumn{2}{|c|}{ Variable } & \multirow[t]{2}{*}{ HR } & \multirow[t]{2}{*}{$P$} & \multirow[t]{2}{*}{$95 \%$ CI } \\
\hline & Observed survival & & & & \\
\hline \multirow[t]{7}{*}{ A } & Age (reference: <65) & $\geq 65$ & 1.37 & .35 & $0.71-2.63$ \\
\hline & Sex (reference: male) & Female & 1.25 & .50 & $0.65-2.38$ \\
\hline & Histology (reference: epithelioid) & Nonepithelioid & 3.77 & .02 & $1.19-11.94$ \\
\hline & Treatment (reference: only chemotherapy) & Chemotherapy and surgery & 0.56 & .13 & $0.26-1.18$ \\
\hline & & Only surgery & 0.92 & .85 & $0.39-2.17$ \\
\hline & & Not treated & 0.36 & .16 & $0.08-1.52$ \\
\hline & & Missing information & 0.60 & .51 & $0.13-2.74$ \\
\hline \multirow[t]{8}{*}{ D } & Age (reference: <65) & $\geq 65$ & 1.37 & .35 & $0.71-2.62$ \\
\hline & Sex (reference: male) & Female & 2.07 & .06 & $0.97-4.41$ \\
\hline & Histology (reference: epithelioid) & Nonepithelioid & 3.53 & .02 & $1.27-9.83$ \\
\hline & Treatment (reference: only chemotherapy) & Chemotherapy and surgery & 0.33 & .01 & $0.14-0.76$ \\
\hline & & Only surgery & 0.65 & .34 & $0.27-1.57$ \\
\hline & & Not treated & 0.36 & .11 & $0.1-1.28$ \\
\hline & & Missing information & 0.69 & .63 & $0.15-3.15$ \\
\hline & TV (reference: less than average) & Greater than average & 3.47 & $<.1$ & $1.5-8.02$ \\
\hline \multirow[t]{9}{*}{$E$} & Age (reference: <65) & $\geq 65$ & 2.61 & .01 & $1.22-5.57$ \\
\hline & Sex (reference: male) & Female & 1.28 & .44 & $0.68-2.42$ \\
\hline & Histology (reference: epithelioid) & Nonepithelioid & 2.99 & .05 & $1.02-8.74$ \\
\hline & Treatment (reference: only chemotherapy) & Chemotherapy and surgery & 0.67 & .30 & $0.32-1.42$ \\
\hline & & Only surgery & 1.46 & .45 & $0.55-3.91$ \\
\hline & & Not treated & 0.54 & .38 & $0.13-2.16$ \\
\hline & & Missing information & 0.24 & .09 & $0.05-1.22$ \\
\hline & NPS (reference: 1) & 2 & 3.00 & .08 & $0.87-10.37$ \\
\hline & & 3 & 6.05 & $<.1$ & $1.91-19.12$ \\
\hline \multirow[t]{12}{*}{$\mathrm{F}$} & Age (reference: <65) & $\geq 65$ & 2.71 & .02 & $1.2-6.12$ \\
\hline & Sex (reference: male) & Female & 1.76 & .13 & $0.85-3.64$ \\
\hline & Histology (reference: epithelioid) & Nonepithelioid & 2.95 & .04 & $1.03-8.43$ \\
\hline & Treatment (reference: only chemotherapy) & Chemotherapy and surgery & 0.45 & .08 & 0.18-1.09 \\
\hline & & Only surgery & 0.99 & .99 & 0.33-3.01 \\
\hline & & Not treated & 0.42 & .22 & $0.11-1.65$ \\
\hline & & Missing information & 0.26 & .11 & $0.05-1.36$ \\
\hline & $\begin{array}{l}\text { TV and NPS (reference: small TV } \\
\text { and } 1 \text { NPS) }\end{array}$ & Small TV and 2 NPS & 8.16 & $<.1$ & $1.97-33.87$ \\
\hline & & Small and 3 NPS & 5.19 & .03 & $1.17-23.07$ \\
\hline & & Large TV and 1 NPS & 9.02 & .03 & $1.24-65.71$ \\
\hline & & Large TV and 2 NPS & 8.98 & .05 & $1.01-79.91$ \\
\hline & & Large TV and 3 NPS & 17.76 & $<.1$ & $3.44-91.8$ \\
\hline
\end{tabular}

Abbreviations: HR = hazard ratio; NPS $=$ number of pleural sites; TV $=$ tumor volume.

prognostic stratification of MPM pts. In that study, the quantitative clinical staging, on the basis of TV combined with the measurement of the maximal fissural thickness at the time of CT imaging, performed statistically better than standard clinical TNM staging (c-index, $0.638[95 \% \mathrm{CI}, 0.603-0.673]$ vs. c-index $=0.562[95 \%$ CI, 0.525-0.599]; $P=.001) .^{24}$

The need for quantitative parameters to better stratify MPM patient prognosis derives from the limits of the TNM staging system in MPM disease, which is characterized by a peculiar morphology with extension to surrounding organs. However, in other malignancies, the definition of the T parameter of the TNM classification is on the basis of the size measurement of the lesions; in MPM T value is a qualitative parameter, expressing the disease relationships with adjacent structures. For these reasons its definition is influenced by the subjective impression of the single radiologist and its value is conditioned by unavoidable inter/intraobserver variability, often with lack of correspondence between clinical and pathological stage. $^{11}$

In our study, the clinical TNM and T value in particular, might have been positively conditioned by the high level of expertise of the MPM-dedicated thoracic radiologists.

We decided to evaluate NPS as a further objective quantitative parameter because MPM macroscopic involvement of 1 or more of the pleural surfaces can usually be easily defined on CT scan. In 


\section{Tumor Volume and Number of Pleural Sites in MPM}

addition, it might be the expression of the greater or smaller local extent of disease that might condition patient prognosis. Our NPS analysis was only on the basis of the number of involved sites. We did not consider which specific pleural surface (parietal, diaphragmatic, or mediastinal) was involved because of the small number of pts included in the study, and the frequent concurrent involvement of 2 or 3 sites; a further stratification was not possible. Larger prospective studies are needed to evaluate the effect of single pleural surface involvement combined with the volume on patient prognosis.

In contrast with actual tendency in MPM management, a high percentage of our pts, almost half, underwent surgery, also in advanced stage. To note, the period of data collection predates the MARS (Mesothelioma and Radical Surgery) study ${ }^{27}$ era. At that time the role of surgery was still considered relevant and not so debated as today, when alternative systemic treatment seems to play a greater role. ${ }^{28}$ In addition, INT is a highly specialized institution for surgery treatment in MPM pts and management (including radiological expertise) and this could contribute to explain the high number of pts surgically treated and diagnosed in advanced stage in our study.

Several limitations exist in this study. First of all, it is a small retrospective analysis. Second, the seventh TNM edition was used to calculate the clinical stage. We are conscious that the eighth edition has been available for $>2$ years, however, we decided to use the previous TNM staging because our study refers to a period that ended when the eighth edition TNM staging did not exist. CT scans used to define TNM staging, TV, and NPS were all evaluated by 2 different thoracic radiologists. A $100 \%$ concordance was reported for NPS evaluation, supporting the objectivity of our new parameter. This indicates that the intraobserver variability was very low in our study.

The CT scans were performed in different diagnostic centers, using different machines with variable resolution, so the heterogeneity in slice thickness might have partially influenced radiological evaluation. The presence of a highly experienced radiologist might have partially conditioned the staging and the definition of the other 2 parameters.

\section{Conclusion}

We confirmed the prognostic role of TNM, T, TV, NPS, and, mostly, the combinations of TV and NPS, highlighting the importance of considering different quantitative parameters to define the prognosis of MPM pts. However, the creation of a new model to define patient prognosis is still far off. The translation of such an approach from the research level to clinical practice might be complex, requiring the application of new radiological methods and new parameters. Moreover, a broad experience on all of these aspects and the standardization of scan resolution and radiological reports will be needed.

The actual TNM staging does not satisfy clinical need for MPM, therefore we believe that combining CT scan quantitative parameters such as TV and NPS could increase prognostic accuracy for MPM pts. Further collaborative studies are needed.

\section{Clinical Practice Points}

- Age, sex, stage, histotype, and surgery are the most recognized prognostic factors for MPM.

- New approaches such as TV and NPS could be useful to improve staging.

- Our study showed that TNM, T, TV, and NPS play a prognostic role.

- The TV and NPS combination performed better than TV, NPS, and TNM alone as a prognostic classifier.

- The combination of different quantitative parameters might increase prognostic accuracy.

\section{Acknowledgments}

The study was funded by the Associazione Italiana per la Ricerca sul Cancro (AIRC) (investigator grant IG 2012 N.13534) and Italian Ministry of Health (Ricerca Finalizzata 2008, RF-20081241451).

\section{Disclosure}

Marina Garassino declares personal financial interests, with AstraZeneca, MSD International GmbH, BMS, Boehringer Ingelheim Italia S.P.A, Celgene, Eli Lilly, Ignyta, Incyte, Inivata, MedImmune, Novartis, Pfizer, Roche, and Takeda; institutional financial interests with Eli Lilly, MSD, Pfizer (MISP); AstraZeneca, MSD International GmbH, BMS, Boehringer Ingelheim Italia S.P.A, Celgene, Eli Lilly, Ignyta, Incyte, Inivata, MedImmune, Novartis, Pfizer, Roche, Takeda, Tiziana, and Foundation Medicine; other research funding from AIRC, AIFA, Italian Moh, and TRANSCAN. The remaining authors have stated that they have no conflicts of interest.

\section{References}

1. Pass H, Giroux D, Kennedy C, et al. The IASLC Mesothelioma Staging Project: improving staging of a rare disease through international participation. IASLC Staging and Prognostic Factors Committee, Advisory Boards and Participating Institutions. J Thorac Oncol 2016; 12:2082-8.

2. Stayner L, Welch LS, Lemen R. The worldwide pandemic of asbestos-related diseases. Annu Rev Public Health 2013; 34:205-16.

3. Tunesi S, Ferrante D, Mirabelli D, et al. Gene-asbestos interaction in malignant pleural mesothelioma susceptibility. Carcinogenesis 2015; 36:1129-35.

4. Robinson BM. Malignant pleural mesothelioma: an epidemiological perspective. Ann Cardiothorac Surg 2012; 1:491-6.

5. Rusch VW, Venkatraman ES. Important prognostic factors in patients with malignant pleural mesothelioma, managed surgically. Ann Thorac Surg 1999; 68: 1799-804.

6. Rusch VW, Chansky K, Kindler HL, et al. The IASLC Mesothelioma Staging Project: Proposals for the $\mathrm{M}$ descriptors and for revision of the TNM stage groupings in the forthcoming (eighth) edition of the TNM Classification for Mesothelioma. I Thorac Oncol 2016; 12:2112-9.

7. Zahid I, Sharif S, Routledge T, et al. What is the best way to diagnose and stage malignant pleural mesothelioma? Interact Cardiovasc Thorac Surg 2011; 2:254-9.

8. Kindler HL, Ismaila N, Armato SG 3rd, et al. Treatment of malignant pleura mesothelioma: American Society of Clinical Oncology clinical practice guideline. J Clin Oncol 2018; 13:1343-73.

9. Scherpereel A, Astoul P, Baas P, et al. Guidelines of the European Respiratory Society and the European Society of Thoracic Surgeons for the management of malignant pleural mesothelioma. Eur Respir J 2010; 35:479-95.

10. Pass HI, Temeck BK, Kranda K, et al. Preoperative tumor volume is associated with outcome in malignant pleural mesothelioma. I Thorac Cardiovasc Surg 1998; 115:310-7, discussion 317-8

11. Gill RR, Richards WG, Yeap BY, et al. Epithelial malignant pleural mesothelioma after extrapleural pneumonectomy: stratification of survival with CT-derived tumor volume. Am J Roentgenol 2012; 198:359-63. 
12. Liu F, Zhao B, Krug LM, et al. Assessment of therapy responses and prediction of survival in malignant pleural mesothelioma through computer-aided volumetric measurement on computed tomography scans. J Thorac Oncol 2010; 5 . 879-84.

13. Prasad SR, Jhaveri KS, Saini S, et al. CT tumor measurement for therapeutic response assessment: comparison of unidimensional, bidimensional, and volumetric techniques-initial observations. Radiology 2002; 225:416-9.

14. Creaney J, Francis RJ, Dick IM, et al. Serum soluble mesothelin concentrations in malignant pleural mesothelioma: relationship to tumor volume, clinical stage and changes in tumor burden. Clin Cancer Res 2011; 17:1181-9.

15. Plathow C, Klopp M, Thieke C, et al. Therapy response in malignant pleura mesothelioma-role of MRI using RECIST, modified RECIST and volumetric approaches in comparison with CT. Eur Radiol 2008; 18:1635-43.

16. Frauenfelder T, Tutic M, Weder W, et al. Volumetry: an alternative to asses therapy response for malignant pleural mesothelioma? Eur Respir J 2011; 38: $162-8$.

17. Cheng L, Tunariu N, Collins DJ, et al. Response evaluation in mesothelioma: beyond RECIST. Lung Cancer 2015; 90:433-41.

18. Labby ZE, Nowak AK, Dignam JJ, et al. Disease volumes as a marker for patient response in malignant pleural mesothelioma. Ann Oncol 2013; 24:999-1005.

19. Labby ZE, Armato SG 3rd, Dignam JJ, et al. Lung volume measurements as a surrogate marker for patient response in malignant pleural mesothelioma. $J$ Thorac Oncol 2013; 8:478-86.

20. Ak G, Metintas M, Metintas S, et al. Three-dimensional evaluation of chemotherapy response in malignant pleural mesothelioma. Eur J Radiol 2010; 74:130-5.
21. Rusch VW, Gill R, Mitchell A, et al. A multicenter study of volumetric computed tomography for staging malignant pleural mesothelioma. Ann Thorac Surg 2016; 102:1059-66

22. Nowak AK, Chansky K, Rice DC, et al. The IASLC Mesothelioma Staging Project: proposals for revisions of the $\mathrm{T}$ descriptors in the forthcoming eighth edition of the TNM classification for pleural mesothelioma. J Thorac Oncol 2017 ; 11:2089-99.

23. de Perrot M, Dong Z, Bradbury P, et al. Impact of tumor thickness on survival after radical radiation and surgery in malignant pleural mesothelioma. Eur Respir J 2017; 49:1601428.

24. Gill RR, Yeap BY, Bueno R, et al. Quantitative clinical staging for patients with malignant pleural mesothelioma. I Natl Cancer Inst 2018; 110:258-64.

25. Gill RR, Naidich DP, Mitchell A, et al; Malignant Mesothelioma Volumetric CT Study Group. North American multicenter volumetric CT study for clinical staging of malignant pleural mesothelioma: feasibility and logistics of setting up a quantitative imaging study. I Thorac Oncol 2016; 11:1335-44.

26. Paajanen J, Laaksonen S, Ilonen I, et al. Computed tomography in the evaluation of malignant pleural mesothelioma-association of tumor size to a sarcomatoid histology, a more advanced TNM stage and poor survival. Lung Cancer 2018; 116:73-9.

27. Treasure T, Lang-Lazdunski L, Waller D, et al. Extra-pleural pneumonectomy versus no extra-pleural pneumonectomy for patients with malignant pleural mesothelioma: clinical outcomes of the Mesothelioma and Radical Surgery (MARS) randomised feasibility study. Lancet Oncol 2011; 12:763-72.

28. Signorelli D, Macerelli M, Proto C, et al. Systemic approach to malignant pleural mesothelioma: what news of chemotherapy, targeted agents and immunotherapy? Tumori 2016; 102:18-30. 


\section{Tumor Volume and Number of Pleural Sites in MPM}

\section{Supplemental Data}

Supplemental Table 1 Differences Between Patients Treated or Not With Radical Surgery, Demographic and Clinical Characteristics
of the Patients According to Radical Surgery, Chemotherapy, and Treatment Received

\begin{tabular}{|c|c|c|c|c|c|}
\hline \multicolumn{3}{|c|}{ Surgically Treated $(\mathrm{PD}+)$} & \multicolumn{2}{|c|}{ Surgically Treated (EPP) } & Not Surgically Treated \\
\hline \multicolumn{6}{|l|}{ Radical Surgery } \\
\hline \multicolumn{2}{|l|}{ Cases, $n$} & 9 & \multicolumn{2}{|l|}{14} & 29 \\
\hline Mean age, y & & 62 & \multicolumn{2}{|l|}{63} & 62 \\
\hline \multicolumn{6}{|l|}{ Histology } \\
\hline Epithelioid & & $89 \%$ & \multicolumn{2}{|l|}{$79 \%$} & $86 \%$ \\
\hline \multicolumn{6}{|l|}{ Sex } \\
\hline Male & & $67 \%$ & $71 \%$ & & $66 \%$ \\
\hline \multicolumn{6}{|l|}{ Stage } \\
\hline \multicolumn{2}{|l|}{ Early } & $11 \%$ & \multicolumn{2}{|l|}{$14 \%$} & $10 \%$ \\
\hline \multicolumn{2}{|l|}{ III } & $33 \%$ & \multicolumn{2}{|l|}{$43 \%$} & $38 \%$ \\
\hline \multicolumn{2}{|l|}{ IV } & $56 \%$ & \multicolumn{2}{|l|}{$33 \%$} & $52 \%$ \\
\hline Chemotherapy & Chen & notherapy Done & No Chemoth & & sing Information \\
\hline Cases, n & & 37 & 13 & & 2 \\
\hline Mean age & & 62 & 64 & & 65 \\
\hline Histology & & & & & \\
\hline Epithelioid & & $92 \%$ & $62 \%$ & & $100 \%$ \\
\hline Sex & & & & & \\
\hline Male & & $65 \%$ & $69 \%$ & & $100 \%$ \\
\hline Stage & & & & & \\
\hline Early & & $11 \%$ & $15 \%$ & & $0 \%$ \\
\hline III & & $35 \%$ & $54 \%$ & & $0 \%$ \\
\hline IV & & $54 \%$ & $31 \%$ & & $100 \%$ \\
\hline Treatment & Only Chemotherapy & $\begin{array}{l}\text { Chemotherapy and } \\
\text { Surgery }\end{array}$ & Only Surgery & Not Treated & Missing Information \\
\hline Cases, $n$ & 24 & 13 & 8 & 5 & 2 \\
\hline Mean age, y & 62 & 61 & 65 & 63 & 65 \\
\hline Histology & & & & & \\
\hline Epithelioid & $96 \%$ & $85 \%$ & $75 \%$ & $40 \%$ & $100 \%$ \\
\hline Sex & & & & & \\
\hline Male & $67 \%$ & $62 \%$ & $75 \%$ & $60 \%$ & $100 \%$ \\
\hline Stage & & & & & \\
\hline Early & $4 \%$ & $23 \%$ & $0 \%$ & $40 \%$ & $0 \%$ \\
\hline III & $38 \%$ & $31 \%$ & $62 \%$ & $40 \%$ & $0 \%$ \\
\hline IV & $58 \%$ & $46 \%$ & $38 \%$ & $20 \%$ & $100 \%$ \\
\hline
\end{tabular}

Abbreviations: EPP = extrapleural pneumonectomy; PD+ = pleurectomy/decortication. 